\title{
Dynamic Response Analysis of High-Pressure Rocket Pumps
}

\author{
By Sang-In Jun, ${ }^{1)}$ Takashi ToKumasu ${ }^{2)}$ and Kenjiro KAMIJO ${ }^{2)}$ \\ ${ }^{1)}$ Graduate School of Engineering, Tohoku University, Sendai, Japan \\ ${ }^{2)}$ Institute of Fluid Science, Tohoku University, Sendai, Japan \\ (Received October 2nd, 2003)
}

\begin{abstract}
With regard to the development of a liquid-fuel rocket engine, knowledge of unsteady characteristics of turbopumps is essential in attempts to increase rocket reliability. Numerical simulation is very advantageous in determining the unsteady characteristics of turbopumps, and knowledge thus obtained can contribute to decreasing the cost and the number of experiments. In the present study, the effect of compressibility of cryogenic propellants, such as liquid hydrogen $\left(\mathrm{LH}_{2}\right)$ and liquid oxygen (LOX), and the effect of nonlinearity of flow on the dynamic response of a high-pressure turbopump were considered. The results of calculation were compared with a nonlinear incompressible mathematical model. The effects of dynamic characteristics of a cavitating inducer, such as cavitation compliance and mass flow gain factor, as well as the effect of pipe elasticity and that of an accumulator for the POGO suppressor, were also analyzed.
\end{abstract}

Key Words: Cavitating Inducer, Dynamic Response, Cryogenic Propellants, Compressibility, Nonlinear Model

\section{Nomenclature}

$A:$ cross-sectional area $\left(\mathrm{m}^{2}\right)$

$S_{a}, S_{b}$ : coefficients of pump performance curve

$C_{B}$ : cavitation compliance $\left(\mathrm{m}^{2}\right)$

$g:$ gravitational acceleration $\left(\mathrm{m} / \mathrm{s}^{2}\right)$

$H$ : enthalpy $(\mathrm{J} / \mathrm{kg})$

$L$ : inertance coefficient $\left(\mathrm{m}^{-1}\right)$

$L_{v}$ : latent heat $(\mathrm{J} / \mathrm{kg})$

$M$ : mass flow rate $(\mathrm{kg} / \mathrm{s})$

$M_{B}$ : mass flow gain factor (s)

$P$ : pressure $(\mathrm{Pa})$

$R:$ resistance coefficient $\left(\mathrm{m}^{-4}\right)$

$T:$ temperature $(\mathrm{K})$

$t:$ time (s)

$U$ : internal energy $(\mathrm{J} / \mathrm{kg})$

$V$ : volume of cell $\left(\mathrm{m}^{3}\right)$

$\alpha$ : pipe elasticity coefficient (m)

$\gamma$ : gas constant

$\rho:$ density $\left(\mathrm{kg} / \mathrm{m}^{3}\right)$

$\rho_{0}$ : density of incompressible flow $\left(\mathrm{kg} / \mathrm{m}^{3}\right)$

Subscripts

1: upstream

2: downstream

A : accumulator

$\mathrm{C}:$ cavity

$\mathrm{O}$ : orifice in throat of an accumulator

$\mathrm{p}$ : pump

$\mathrm{T}$ : throat of an accumulator

v : vapor

Superscripts

* : branch of a cell

\section{Introduction}

During a launch sequence, a rocket engine experiences many transient stages such as chilling and engine start, thrust control, engine shutdown and reignition, etc. Stable operation of an engine system during these stages is a very important design criterion for rocket engine reliability, so unsteady performance tests constitute the major part of rocket engine tests. Numerical simulation enables us to determine the unsteady behavior of the system and helps to make it possible to decrease the cost and number of tests.

The turbopump is a key component of liquid rocket engines, which makes high-pressure combustion possible. To build a high-performance rocket requires a high-pressure turbopump that is both compact and light. To achieve these characteristics, such a turbopump must rotate at a very high speed. An inducer is attached to the front of the main impeller to prevent cavitation from occurring because cavitation results in reducing pump suction performance. However, cavitation also occurs in the inlet section of the inducer because very high suction performance is needed for a highpressure turbopump. The dynamic characteristics of cavitation are determined by the design of the inducer and are known to be very critical and complex factors in the stability of a rocket engine. They can cause considerable vibration and oscillation problems in the propellant feed system. Rotating cavitation and cavitation surge, which are the main problems in rocket pumps, are typical examples.

Brennen and Acosta ${ }^{1-4)}$ have derived turbopump characteristics parameters, cavitation compliance $\left(C_{B}\right)$ and mass flow gain factor $\left(M_{B}\right)$, and Tsujimoto, Kamijo and Shimura $^{5-10)}$ have analyzed the effects of these parameters theoretically and experimentally. The cavitation compliance $\left(C_{B}\right)$ is defined as the ratio of the cavity volume change rate to the pump inlet pressure change rate, and the mass flow 
gain factor $\left(M_{B}\right)$ is defined as the ratio of the cavity volume change rate to the change rate of the pump inlet flow rate. Since these parameters are particularly important in stability analysis of rocket engines, considerable efforts have been made to analyze and evaluate them. However, quantitatively applicable analyses or design criteria for turbopumps have not been available because there is significant nonlinearity in real systems and, moreover, because cryogenic propellants such as $\mathrm{LH}_{2}$ and LOX are characterized by compressibility. Most analytical models to date have been based on incompressible mathematical models, which cannot accurately simulate the flow of highly compressible propellant such as $\mathrm{LH}_{2}$. Many are modeled by linear equations that are valid only for small disturbances.

This paper analyzes the effect of the compressibility of cryogenic propellants, such as $\mathrm{LH}_{2}$ and LOX, on the dynamic response of pumping systems. Pumping systems of the LE-7 engine, including pipes, were modeled by the one-dimensional nonlinear compressible mathematical model and the simulation results of dynamic response to tank pressure drop or pump outlet blowdown pressure drop were compared with those of a nonlinear incompressible mathematical model. Effects of cavitation compliance and the mass flow gain factor on system stability were quantitatively analyzed by spectral analysis of the time-dependent simulation results.

\section{Mathematical Formulation}

\subsection{Compressible flow model}

\subsubsection{Analysis model}

Figures 1(a) and 1(b) show schematic of the $\mathrm{LH}_{2}$ feed system and the LOX feed system, respectively, of the LE7 engine. Each is composed of a propellant tank, a pump including an inducer, and inlet and outlet pipes. These systems were modeled as one-dimensional flow circuits and the $\mathrm{LH}_{2}$ and LOX pumps were modeled as tapered pipes with lengths of $7.2 \mathrm{~m}$ and $2.5 \mathrm{~m}$, respectively. Figure 2 shows a schematic of nodes and branches. The inlet pipe, pump and outlet pipe are divided into $L, M$ and $N$ cells, respectively. Each

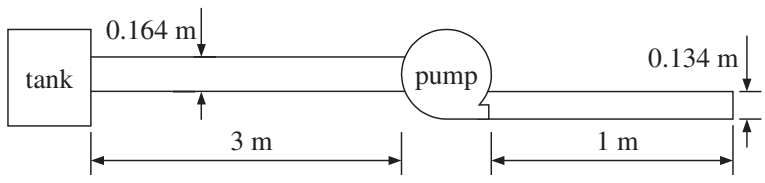

(a) $\quad \mathrm{LH}_{2}$ feed system

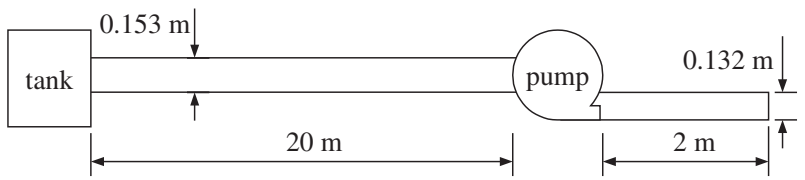

(b) LOX feed system

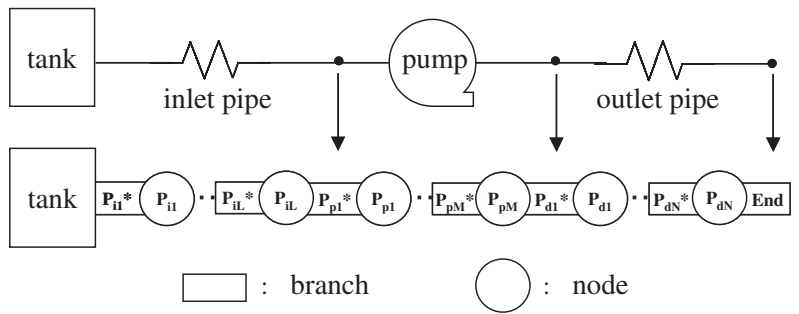

Fig. 2. Schematic for compressible flow model.

cell is composed of a node and a branch. Mass and energy are conserved in each node and momentum is conserved in each branch.

\subsubsection{Pipe model}

A rocket engine feed system can be considered as a piping system and a pipe model is the basis of each component. The following equations model the pipe.

The mass conservation equation, momentum conservation equation and energy conservation equation are

$$
\begin{aligned}
& V_{k} \frac{\mathrm{d} \rho_{k}}{\mathrm{~d} t}=M_{k}^{*}-M_{k+1}^{*} \\
& P_{k-1}+\frac{1}{2} \frac{M_{k-1}^{2}}{\rho_{k-1} A_{k-1}^{2}}-P_{k}-\frac{1}{2} \frac{M_{k}^{2}}{\rho_{k} A_{k}^{2}} \\
& =R_{k} \frac{M_{k}^{* 2}}{\rho_{k}^{*}}+L_{k} \frac{\mathrm{d} M_{k}^{*}}{\mathrm{~d} t}
\end{aligned}
$$

and

$$
\begin{aligned}
\frac{\mathrm{d}}{\mathrm{d} t}[ & \left.\rho_{k} V_{k}\left(U_{k}+\frac{1}{2} \frac{M_{k}^{2}}{\rho_{k}^{2} A_{k}^{2}}\right)\right] \\
= & M_{k}^{*}\left(H_{k}^{*}+\frac{1}{2} \frac{M_{k}^{* 2}}{\rho_{k}^{* 2} A_{k}^{* 2}}\right) \\
& -M_{k+1}^{*}\left(H_{k+1}^{*}+\frac{1}{2} \frac{M_{k+1}^{*} \rho_{k+1}^{*} A_{k}^{* 2}}{2}\right)
\end{aligned}
$$

The relation for the cross-sectional areas is

$$
A_{k}=\frac{A_{k}^{*}+A_{k+1}^{*}}{2}
$$

The equation of state is defined in a branch and Van der Waals equation was used because calculation of steady-state conditions is difficult for the equation of state for an ideal gas. The parameters $a, b$, and $R$ were obtained so the equation is consistent with the physical properties of $\mathrm{LH}_{2}$ and LOX used in the LE-7 engine. The Van der Waals equation is

$$
\left(P_{k}^{*}+a \rho_{k}^{* 2}\right)\left(\frac{1}{\rho_{k}^{*}}-b\right)=R T_{k}^{*}
$$

The pressure, temperature and mass flow rate of each branch were averaged to obtain values for each intermediate node:

Fig. 1. Schematics of analysis model. 


$$
\begin{aligned}
& P_{k}=\frac{P_{k}^{*}+P_{k+1}^{*}}{2} \\
& T_{k}=\frac{T_{k}^{*}+T_{k+1}^{*}}{2}
\end{aligned}
$$

and

$$
M_{k}=\frac{M_{k}^{*}+M_{k+1}^{*}}{2}
$$

The density of each node was averaged to obtain the values for each intermediate branch:

$$
\rho_{k}^{*}=\frac{\rho_{k-1}+\rho_{k}}{2}
$$

\subsubsection{Pump model}

The mass conservation equation for the pump model has the same shape as Eq. (1). For the momentum conservation equation, the pump is divided into many cells and the pressure rise of each cell is assumed to be proportional to the mass flow rate of each cell. The slope of the performance curve is assumed to be the same as that of the inducer of the LE-7 engine:

$$
\begin{gathered}
P_{k-1}+\frac{1}{2} \frac{M_{k-1}^{2}}{\rho_{k-1} A_{k-1}{ }^{2}}-P_{k}-\frac{1}{2} \frac{M_{k}^{2}}{\rho_{k} A_{k}^{2}} \\
=-S_{a} M_{k}^{*}-S_{b} \rho_{k}^{*}+L_{p} \frac{\mathrm{d} M_{k}^{*}}{\mathrm{~d} t}
\end{gathered}
$$

where, $S_{a}$ and $S_{b}$ are assumed to be $-98000 \mathrm{~m}^{-1} \mathrm{~s}^{-1}$ and $56700 \mathrm{~m}^{2} \mathrm{~s}^{-2}$, respectively.

The energy conservation equation is

$$
\begin{aligned}
\frac{\mathrm{d}}{\mathrm{d} t} & {\left[\rho_{k} V_{k}\left(U_{k}+\frac{1}{2} \frac{M_{k}^{2}}{\rho_{k}^{2} A_{k}^{2}}\right)\right] } \\
= & M_{k}^{*}\left(H_{k}^{*}+\frac{1}{2} \frac{M_{k}^{* 2}}{\rho_{k}^{* 2} A_{k}^{* 2}}\right) \\
& -M_{k+1}^{*}\left(H_{k+1}^{*}+\frac{1}{2} \frac{M_{k+1}^{*}{ }^{2}}{\rho_{k+1}^{*} A_{k+1}^{*}}\right)+Q_{k}
\end{aligned}
$$

where, $Q_{k}$, the required work for a pump, is calculated as

$$
Q_{k}=\left(P_{k+1}^{*}+\frac{1}{2} \frac{M_{k+1}^{*}{ }^{2}}{\rho_{k+1}^{*} A_{k+1}^{*}{ }^{2}}-P_{k}^{*}-\frac{1}{2} \frac{M_{k}^{* 2}}{\rho_{k}^{*} A_{k}^{* 2}}\right) \times \frac{M_{k}}{\rho_{k}}
$$

\subsubsection{Cavitation model}

During a rocket launch sequence, the inducer is under cavitating conditions. The following cavitation equations are applied to the last cell of the inlet pipe.

The mass conservation equation and the energy conservation equation are

$$
\left(V_{k}-V_{\mathrm{C}}\right) \frac{\mathrm{d} \rho_{k}}{\mathrm{~d} t}-\rho_{k} \frac{\mathrm{d} V_{\mathrm{C}}}{\mathrm{d} t}+\rho_{\mathrm{v}} \frac{\mathrm{d} V_{\mathrm{C}}}{\mathrm{d} t}=M_{k}^{*}-M_{k+1}^{*}
$$

and

$$
\begin{aligned}
\frac{\mathrm{d}}{\mathrm{d} t} & {\left[\rho_{k} V_{k}\left(U_{k}+\frac{1}{2} \frac{M_{k}^{2}}{\rho_{k}^{2} A_{k}^{2}}\right)\right]+L_{\mathrm{v}} \rho_{\mathrm{v}} \frac{\mathrm{d} V_{\mathrm{C}}}{\mathrm{d} t} } \\
& =M_{k}^{*}\left(H_{k}^{*}+\frac{1}{2} \frac{M_{k}^{* 2}}{\rho_{k}^{* 2} A_{k}^{* 2}}\right)
\end{aligned}
$$

$$
-M_{k+1}^{*}\left(H_{k+1}^{*}+\frac{1}{2} \frac{M_{k+1}^{*}{ }^{2}}{\rho_{k+1}^{*}{ }^{2} A_{k+1}^{2}}\right)
$$

where, $\rho_{\mathrm{v}}$, the density in the cavity, is assumed to be constant.

The cavity volume variation is not only a function of the pump inlet pressure variation but also a function of the pump inlet mass flow rate variation. The equation for dynamic characteristics of cavitation is

$$
\frac{\mathrm{d} V_{\mathrm{C}}}{\mathrm{d} t}=-C_{B} \frac{\mathrm{d}}{\mathrm{d} t}\left(\frac{P_{k}}{\rho_{k} g}\right)-M_{B} \frac{\mathrm{d}}{\mathrm{d} t}\left(\frac{M_{k}^{*}}{\rho_{k}^{*}}\right)
$$

where, $C_{B}$ is the cavitation compliance and $M_{B}$ is the mass flow gain factor. Since there is no data available on $\mathrm{LH}_{2}$ and LOX, the data calculated for water, such as $C_{B}^{*}$ and $M_{B}^{*}$, by Brennen and Acosta ${ }^{1)}$ were used after dimensionalization ${ }^{9)}$ as follows:

$$
\overline{C_{B}}=2 g \pi d A_{i} /\left(n u_{t}^{2}\right) \times C_{B}^{*}
$$

and

$$
\overline{M_{B}}=\pi d /\left(n u_{t}\right) \times M_{B}^{*}
$$

where, $d, A_{i}, n$, and $u_{t}$ are inducer diameter, inducer inlet area, number of blades and the inducer tip speed, respectively. For the steady-state condition, with a cavitation number of 0.044 , and flow coefficient of $0.08, C_{B}^{*}$ and $M_{B}^{*}$ have values of 0.6 and 0.08 , respectively. Values used for $d, A_{i}, n$, and $u_{t}$ were $0.164 \mathrm{~m}, 0.021 \mathrm{~m}^{2}, 3$, and $42,000 \mathrm{rpm}$, respectively.

\subsection{Pipe elasticity model}

In Eq. (1) as well as in Eq. (13), the effect of pipe elasticity is not included and the cell volume is assumed to be constant. In section 4.3, we analyze the effect of pipe elasticity. The analysis model for pipe elasticity is described here.

The pipe elasticity coefficient, $\alpha$, is defined as the volume change rate corresponding to the change rate of the pressure head for unit length. For the $\mathrm{LH}_{2}$ feed system, it is assumed to be $3.3382 \times 10^{-8} \mathrm{~m}$ and the same value was used for the LOX feed system. Using the definition of the pipe elasticity coefficient, the volume variation of each cell can be calculated as

$$
\frac{\mathrm{d} V_{k}}{\mathrm{~d} t}=\alpha \frac{l_{k}}{g} \frac{\mathrm{d}}{\mathrm{d} t}\left(\frac{P_{k}}{\rho_{k}}\right)
$$

where $l_{k}$ is the length of a cell. Therefore, to include the effect of pipe elasticity, Eq. (18) must be included in Eqs. (1) and (13).

\subsection{Accumulator model}

Figure 3 shows a schematic for an accumulator for the LOX feed system of the LE-7 engine. Helium gas is charged in the accumulator and LOX flows in and out through orifices installed in the throat.

The helium gas phase change is assumed to be adiabatic

$$
P_{\mathrm{A}} V_{\mathrm{A}}^{\gamma}=\mathrm{const}
$$

where $\gamma$ is the gas constant of helium with a value of 1.667 .

For the throat flow, we assumed a liquid phase flow be- 


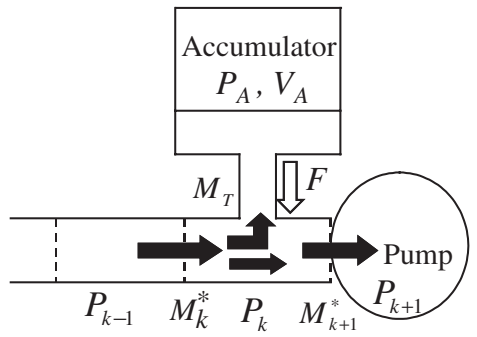

Fig. 3. Schematic of an accumulator

cause the cavity volume is very small compared to the cell volume. The mass flow rate through the throat, $M_{\mathrm{T}}$, was defined as positive when LOX flows into the accumulator and negative when LOX flows out of the accumulator. The mass flow rate through each orifice, $M_{\mathrm{O}}$, is calculated as

$$
M_{\mathrm{O}}=\frac{M_{\mathrm{T}}}{Z}
$$

where $Z$ is the number of orifices

The friction force of each orifice, $F_{\mathrm{O}}$, was calculated as

$$
F_{\mathrm{O}}=\frac{1}{C_{\mathrm{d}}^{2}} \frac{1}{2} \frac{M_{\mathrm{O}}^{2}}{\rho_{k} A_{k}^{2}}
$$

where $C_{\mathrm{d}}$ is the discharge coefficient of the orifice and is assumed to be 0.6. For more detailed analysis, other effects on the momentum loss must be considered. However, they were assumed to be very small and included in the discharge coefficient.

The momentum conservation equation through each orifice is

$$
L_{\mathrm{O}} \frac{\mathrm{d} M_{\mathrm{O}}}{\mathrm{d} t}=P_{k}-P_{\mathrm{A}}-F_{\mathrm{O}} \quad\left(M_{\mathrm{O}}>0\right)
$$

and

$$
L_{\mathrm{O}} \frac{\mathrm{d} M_{\mathrm{O}}}{\mathrm{d} t}=P_{k}-P_{\mathrm{A}}-F_{\mathrm{O}} \quad\left(M_{\mathrm{O}}<0\right)
$$

The mass conservation equation and energy conservation equation of the cell with an installed accumulator are

$$
V_{k} \frac{\mathrm{d} \rho_{k}}{\mathrm{~d} t}=M_{k}^{*}-M_{\mathrm{p}}^{*}-M_{\mathrm{T}}
$$

and

$$
\begin{aligned}
\frac{\mathrm{d}}{\mathrm{d} t}\left[\rho_{k} V_{k}\left(U_{k}+\frac{1}{2} \frac{M_{k}^{2}}{\rho_{k}^{2} A_{k}^{2}}\right)\right] \\
=M_{k}^{*}\left(H_{k}^{*}+\frac{1}{2} \frac{M_{k}^{* 2}}{\rho_{k}^{* 2} A_{k}^{* 2}}\right) \\
\quad-M_{k+1}^{*}\left(H_{k+1}^{*}+\frac{1}{2} \frac{M_{k+1}^{*} \rho_{k+1}^{*} A_{k+1}^{*}}{\rho^{2}}\right) \\
\quad-M_{\mathrm{T}}\left(H_{\mathrm{T}}+\frac{1}{2} \frac{M_{\mathrm{O}}^{2}}{\rho_{k}{ }^{2} A_{\mathrm{O}}{ }^{2}}\right)
\end{aligned}
$$

where, $H_{\mathrm{T}}$, the enthalpy of the orifice flow, is calculated as

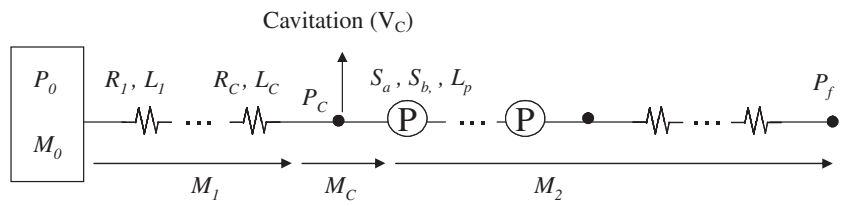

Fig. 4. Schematic of incompressible flow model.

$$
H_{\mathrm{T}}=\frac{H_{k}^{*}+H_{k+1}^{*}}{2}
$$

\subsection{Incompressible flow model}

Figure 4 shows a schematic for the incompressible flow model. For non-cavitating incompressible flow, the mass flow rate varies with time but does not vary with space. The only parameter that varies with both time and space is pressure, and only the momentum conservation equation is used for the governing equation. It is possible to consider only node for each cell in the incompressible flow model. However, for the cavitation model, the mass flow rate becomes discontinuous due to the cavity and another mass conservation equation is needed.

The momentum conservation equations for the inlet and outlet pipes are

$$
P_{k-1}-P_{k}=R_{k} \frac{M_{1}^{2}}{\rho_{0}}+L_{k} \frac{\mathrm{d} M_{1}}{\mathrm{~d} t}
$$

and

$$
P_{k-1}-P_{k}=R_{k} \frac{M_{2}^{2}}{\rho_{0}}+L_{k} \frac{\mathrm{d} M_{2}}{\mathrm{~d} t}
$$

where $M_{1}$ and $M_{2}$ are the mass flow rate upstream and downstream of the cavity, respectively.

The momentum conservation equation for the pump is

$$
\begin{gathered}
P_{k-1}+\frac{1}{2} \frac{M_{2}^{2}}{\rho_{0} A_{k-1}{ }^{2}}-P_{k}-\frac{1}{2} \frac{M_{2}^{2}}{\rho_{0} A_{k}^{2}} \\
=-S_{a} M_{2}-S_{b} \rho_{0}+L_{p} \frac{\mathrm{d} M_{2}}{\mathrm{~d} t}
\end{gathered}
$$

The mass conservation equation and the equation for the dynamic characteristics of cavitation are

$$
\rho_{0} \frac{\mathrm{d} V_{\mathrm{C}}}{\mathrm{d} t}=M_{2}-M_{1}
$$

and

$$
\rho_{0} \frac{\mathrm{d} V_{\mathrm{C}}}{\mathrm{d} t}=-\frac{C_{B}}{g} \frac{\mathrm{d} P_{\mathrm{C}}}{\mathrm{d} t}-M_{B} \frac{\mathrm{d} M_{1}}{\mathrm{~d} t}
$$

where $P_{\mathrm{C}}$ is the pressure of the cell where cavitation occurs.

For cells that are immediately upstream and downstream of the cavity, the momentum conservation equations are

$$
P_{1}+\frac{1}{2} \frac{M_{1}^{2}}{\rho_{0} A_{1}^{2}}-P_{\mathrm{C}}-\frac{1}{2} \frac{M_{\mathrm{C}}{ }^{2}}{\rho_{0} A_{1}{ }^{2}}=R_{\mathrm{C}} \frac{M_{1}^{2}}{\rho_{0}}+L_{\mathrm{C}} \frac{\mathrm{d} M_{1}}{\mathrm{~d} t}
$$

and

$$
P_{\mathrm{C}}+\frac{1}{2} \frac{M_{\mathrm{C}}^{2}}{\rho_{0} A_{1}^{2}}-P_{2}-\frac{1}{2} \frac{M_{2}^{2}}{\rho_{0} A_{2}^{2}}
$$




$$
=-S_{a} M_{2}-S_{b} \rho_{0}+L_{\mathrm{p}} \frac{\mathrm{d} M_{2}}{\mathrm{~d} t}
$$

where, $R_{\mathrm{C}}$ and $L_{\mathrm{C}}$ are the resistance coefficient and the inertance coefficient, respectively, for the cell where cavitation occurs.

$M_{\mathrm{C}}$ is calculated as

$$
M_{\mathrm{C}}=\frac{M_{1}+M_{2}}{2}
$$

\section{Solution Method}

As mentioned above, no data is available for $C_{B}$ and $M_{B}$ of cryogenic propellants. Therefore, the calculated values of $\overline{C_{B}}$ and $\overline{M_{B}}$ in Eqs. (16) and (17) were used as reference values. However, these values are calculated from water and do not incorporate the effect of compressibility. Therefore, they were multiplied by scaling factors and stability analyses were conducted.

Simulations were conducted for two cases: when tank pressure suddenly drops by $5 \%$ and when pump outlet blowdown pressure suddenly drops by $5 \%$. The inlet boundary is the tank and the outlet boundary is the branch of the last cell of the outlet pipe. The pressures of the inlet boundary and outlet boundary were used as input variables. The following equation was used for the pressure disturbance:

$$
\mathrm{d} P=\mathrm{d} P_{f} \exp \left(-\frac{a}{t}\right)
$$

where $\mathrm{d} P_{f}$ represents the magnitude of the disturbance and $a$ is a coefficient determining the change rate of the disturbance and assumed to be $5 \times 10^{-4} \mathrm{~s}$.

The temperature, density and mass flow rate of the tank remain constant and Neumann conditions are applied to the temperature of the inlet boundary and the density of the outlet boundary, respectively. The resistance coefficient of each cell was determined so that the steady-state conditions were consistent with experimental data for the LE-7 engine. The inertance coefficient of each cell was calculated taking the system geometry into consideration. Unsteady conditions were calculated using the 4th order Runge-Kutta method until $1 \mathrm{~s}$ for the $\mathrm{LH}_{2}$ feed system and $5 \mathrm{~s}$ for the LOX feed system.

For calculation of compressible flow, the result is very sensitive to the number of divisions of the inlet pipe. When there are 2 divisions, the result is at a low frequency, but when there are more than 2 , the result is at a high frequency and it is impossible to calculate the unstable dynamic response. However, the low frequency values were similar to that for the case of 2 division. Consequently, we analyzed only the low-frequency result by limiting the number of divisions of the inlet pipe to 2 . The result was also sensitive to the number of divisions of the pump, and system stability dropped as the number increased. Consequently, we divided the two systems into the same number of divisions, namely, 2, 38 and 2 for the inlet pipe, pump and outlet pipe, respectively.

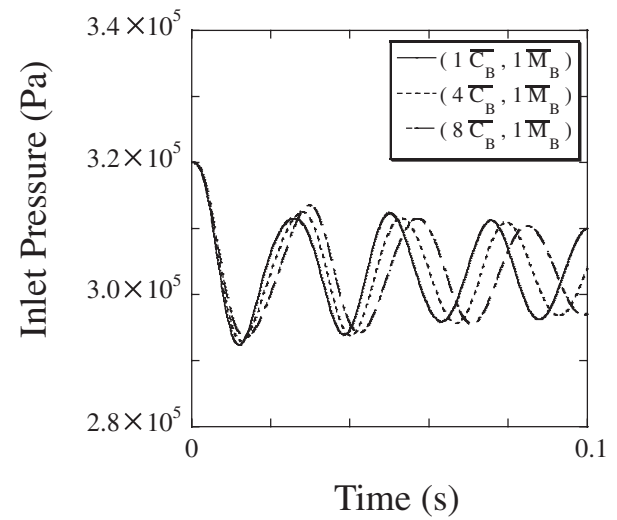

(a) $C_{B}$ effect on $\mathrm{LH}_{2}$ feed system (compressible)

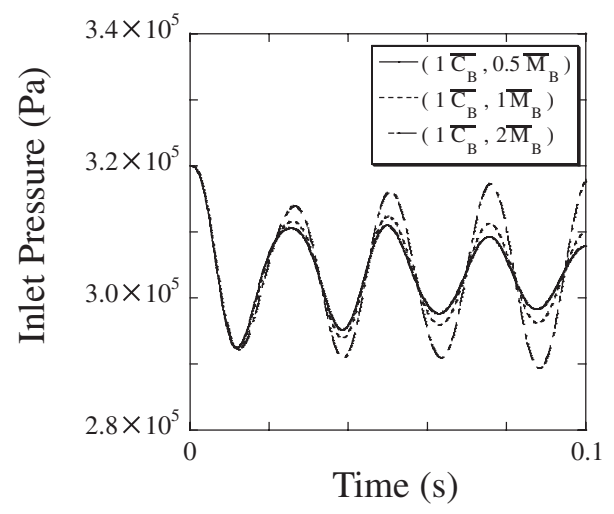

(b) $M_{B}$ effect on $\mathrm{LH}_{2}$ feed system (compressible)

Fig. 5. Effect of $C_{B}$ and $M_{B}$.

\section{Results and Discussion}

\subsection{Effect of cavitation compliance $\left(C_{B}\right)$ and mass flow gain factor $\left(M_{B}\right)$}

Figure 5 shows the effects of $C_{B}$ and $M_{B}$ on the dynamic response of the $\mathrm{LH}_{2}$ feed system. As $C_{B}$ becomes large, the dynamic response frequency becomes low and the dynamic response tends to become stable. As $M_{B}$ becomes large, the dynamic response amplitude becomes large and system stability becomes low.

Figures 6(a) and 6(b) show the neutral stability line and dynamic response frequencies of the $\mathrm{LH}_{2}$ feed system for two flows: compressible, and incompressible. Figures 6(c) and $6(\mathrm{~d})$ show the neutral stability line and dynamic response frequencies of the LOX feed system for two flows: compressible and incompressible. $C_{B}$ is scaled from $0.01 \overline{C_{B}}$ to $10 \overline{C_{B}}$ and $M_{B}$ is scaled from $0.01 \overline{M_{B}}$ to $10 \overline{M_{B}}$. The neutral stability line is nearly longitudinal and lines of equal frequencies are almost horizontal. From these results, we see that $C_{B}$ has a dominant effect on the dynamic response frequency and $M_{B}$ has a dominant effect on system stability. The effect of $C_{B}$ is also large for the stability of the incompressible model.

\subsection{Effect of compressibility}

From Fig. 6, we can see that the results of the compressible model are more stable and have lower frequency re- 


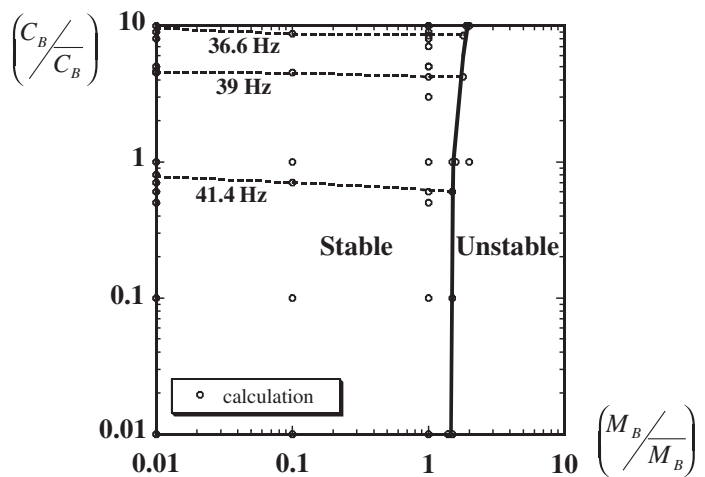

(a) $\mathrm{LH}_{2}$ feed system of compressible model

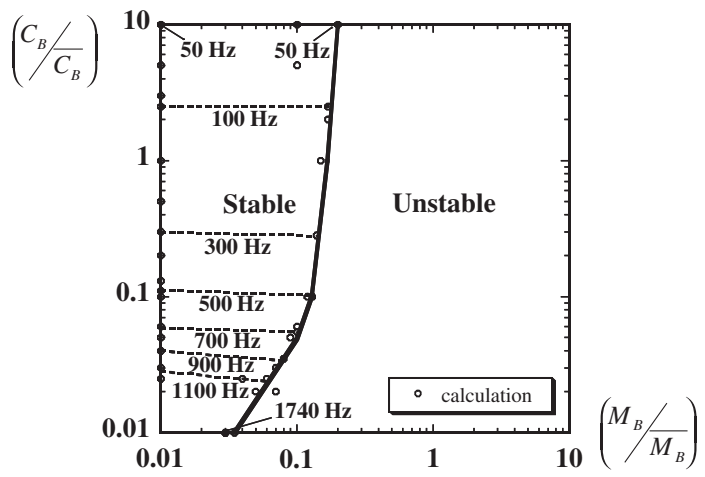

(b) $\mathrm{LH}_{2}$ feed system of incompressible model

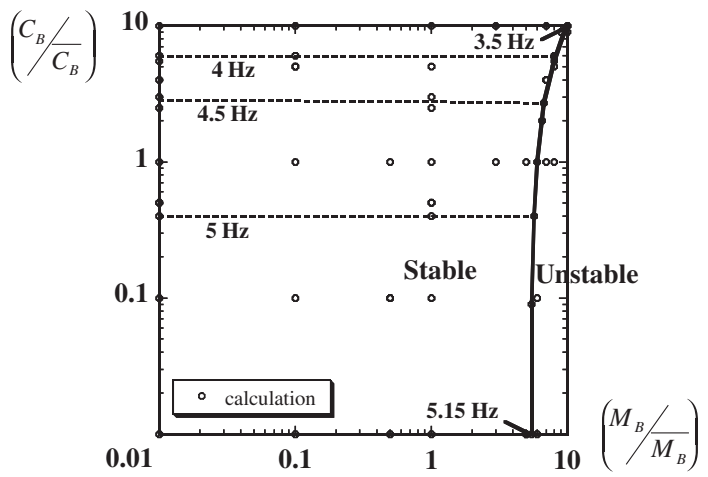

(c) LOX feed system of compressible model

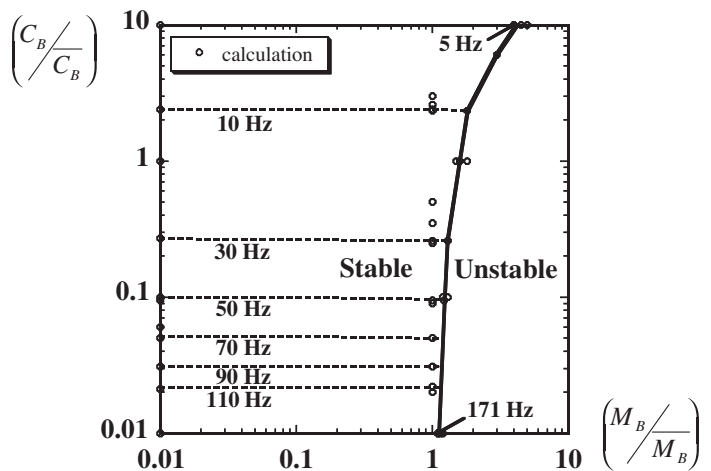

(d) LOX feed system of incompressible model

Fig. 6. Neutral stability line and dynamic response frequency for tank pressure drop of $5 \%$.

sponses than those of the incompressible model. The LOX feed system is more stable than the $\mathrm{LH}_{2}$ feed system because its inlet pipe is more than 6 times longer than that of the $\mathrm{LH}_{2}$ (a)
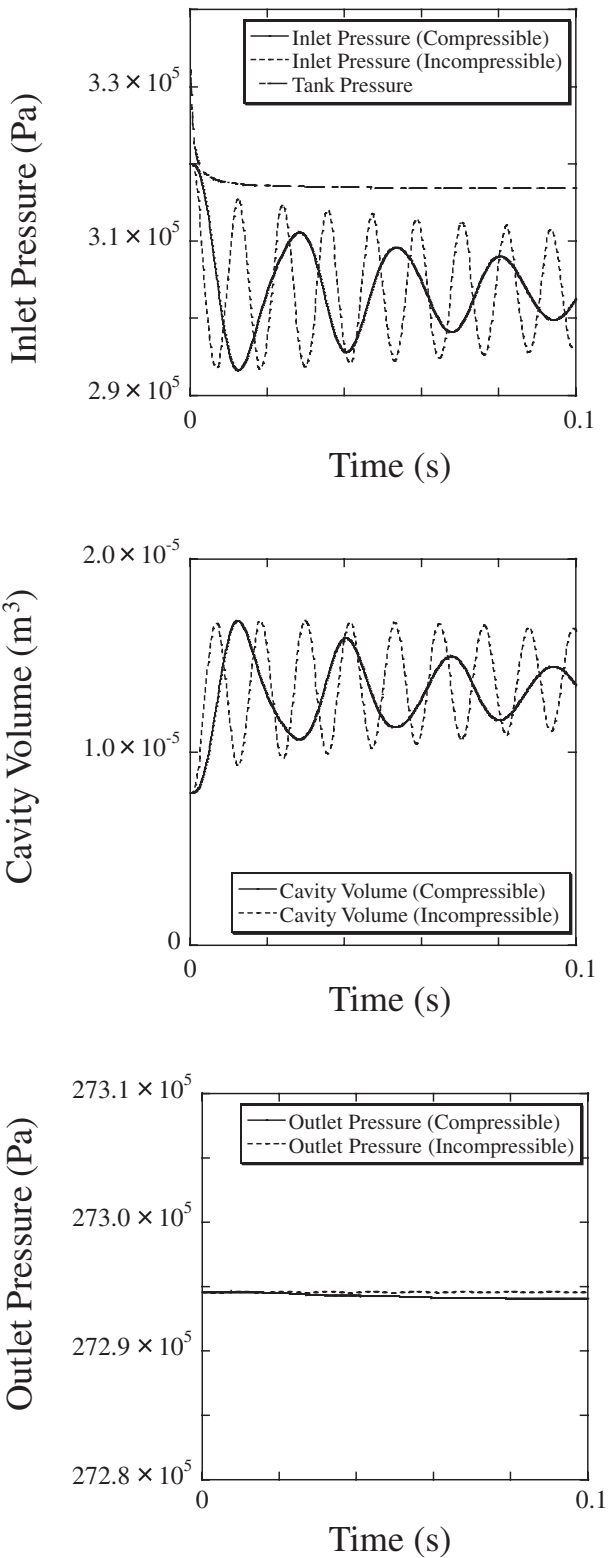

Fig. 7. Dynamic response of $\mathrm{LH}_{2}$ feed system to tank pressure drop of $5 \%\left(5 \overline{C_{B}}, 0.1 \overline{M_{B}}\right)$.

feed system. The system response frequency becomes low as $C_{B}$ becomes large and the frequency of the incompressible model changes more rapidly, with respect to $C_{B}$ change, than that of the compressible model. By comparing the frequency values of the compressible model and the incompressible model, we can see that the dynamic response of the compressible model with a small $C_{B}$ is similar to that of the incompressible model with a large $C_{B}$. As a result, we can say that the compressibility effect is similar to the $C_{B}$ effect.

For the dynamic response frequency of the LE-7 engine, some test results showed $21.88 \mathrm{~Hz}$ for the $\mathrm{LH}_{2}$ feed system and about $4 \mathrm{~Hz}$ for the LOX feed system. ${ }^{11)}$ Consequently, it seems reasonable to analyze the effect of compressibility focusing on the dynamic behavior of the $\mathrm{LH}_{2}$ feed system and the LOX feed system having $C_{B}$ and $M_{B}$ values $5 \overline{C_{B}}$ and $0.1 \overline{M_{B}}$, respectively. Figure 7 shows the dynamic response 
(a)

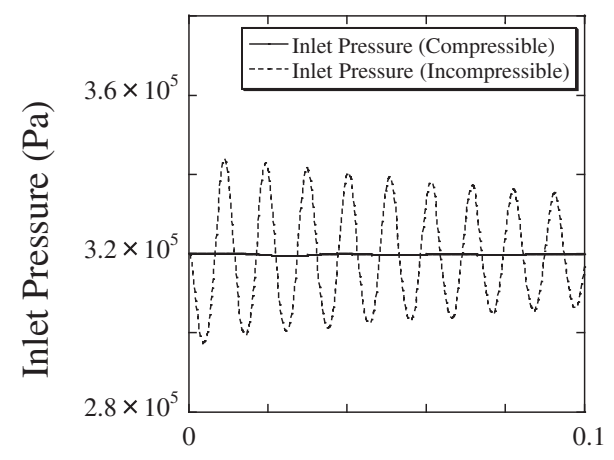

Time (s)

(b)

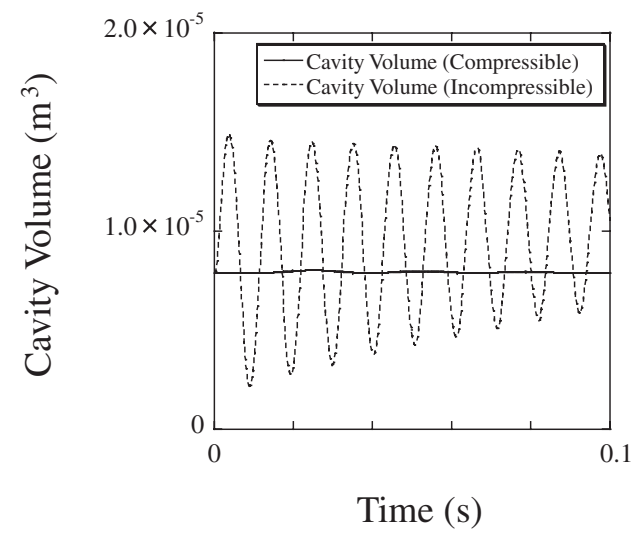

(c)

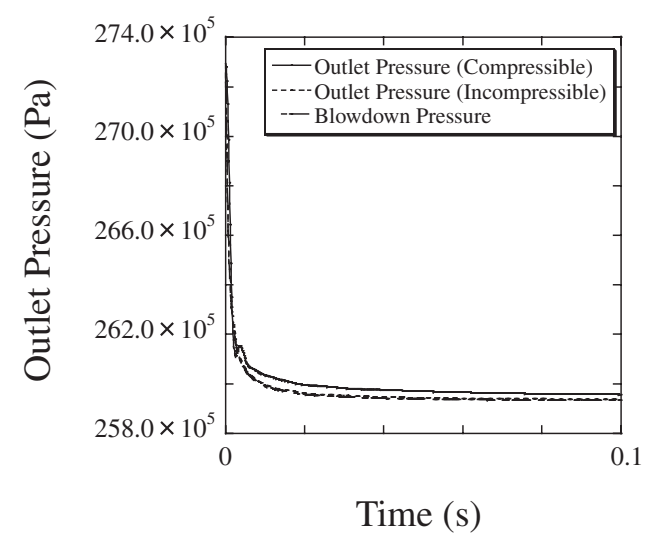

Fig. 8. Dynamic response of $\mathrm{LH}_{2}$ feed system to pump outlet blowdown pressure drop of $5 \%\left(5 \overline{C_{B}}, 0.1 \overline{M_{B}}\right)$.

of the $\mathrm{LH}_{2}$ feed system with $5 \overline{C_{B}}$ and $0.1 \overline{M_{B}}$ to a tank pressure drop of 5\%. Figures 7(a), 7(b), and 7(c) show pump inlet pressure variation, cavity volume variation, and pump outlet pressure variation, respectively. The response frequency of the compressible model is lower than that of the incompressible model and pressure oscillation is rapidly damped.

Figure 8 shows the dynamic response of the $\mathrm{LH}_{2}$ feed system to a pump outlet blowdown pressure drop of 5\%. Figures $8(\mathrm{a}), 8(\mathrm{~b})$, and $8(\mathrm{c})$ show pump inlet pressure variation, cavity volume variation, and pump outlet pressure variation, respectively. In Fig. 7(a), the amplitude ratio of the compressible model to the incompressible model is near 1 , but in Fig. 8(a), the ratio is much lower than 1. This means that the compressibility effect is more dominant for pressure

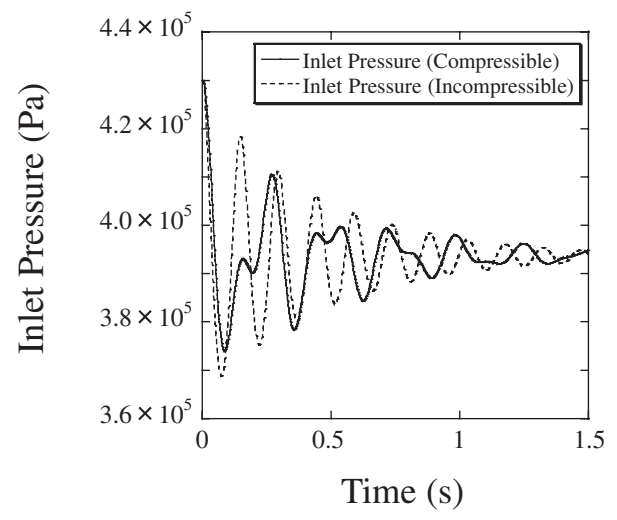

(a) Inlet pressure response to tank pressure drop of $5 \%\left(5 \overline{C_{B}}, 0 . \overline{M_{B}}\right)$

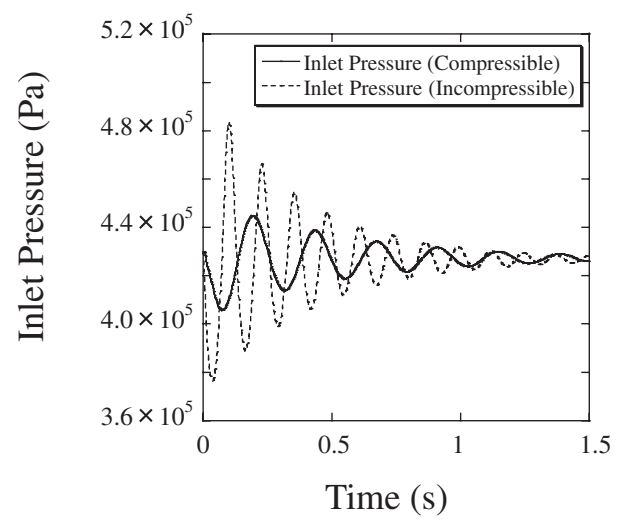

(b) Inlet pressure response to pump outlet blowdown pressure drop of $5 \%\left(5 \overline{C_{B}}, 0.1 \overline{M_{B}}\right)$

Fig. 9. Dynamic response of LOX feed system.

fluctuation downstream of the pump than for pressure fluctuation upstream of the pump. For the compressible model, disturbance downstream of the pump only slightly affected the flow upstream of the pump. The reason seems to be that disturbance energy downstream of the pump is absorbed into the pump by $\mathrm{LH}_{2}$ compressibility.

Figure 9(a) shows the inlet pressure response of the LOX feed system to a tank pressure drop of 5\% and Fig. 9(b) shows the inlet pressure response of the LOX feed system to a pump outlet blowdown pressure drop of $5 \%$. We see that the effect of compressibility of the LOX feed system is similar to that of the $\mathrm{LH}_{2}$ feed system. However, that effect is small compared to that of $\mathrm{LH}_{2}$ feed system as can be seen by comparing the amplitude ratio of the compressible model to incompressible model as shown in Figs. 8(a) and 9(b).

\subsection{Effect of pipe elasticity}

Figure 10(a) shows the effect of pipe elasticity on the $\mathrm{LH}_{2}$ feed system and Fig. 10(b) shows the effect on the LOX feed system. System oscillation becomes slow when pipe elasticity is taken into consideration. By comparing Fig. 5(a) with Fig. 10(a), we see that the effect of pipe elasticity is very similar to the effect of cavitation compliance.

\subsection{Effect of an accumulator}

Figure 11 shows the inlet pressure response of the LOX feed system to a tank pressure drop of 5\% assuming $C_{B}$ 


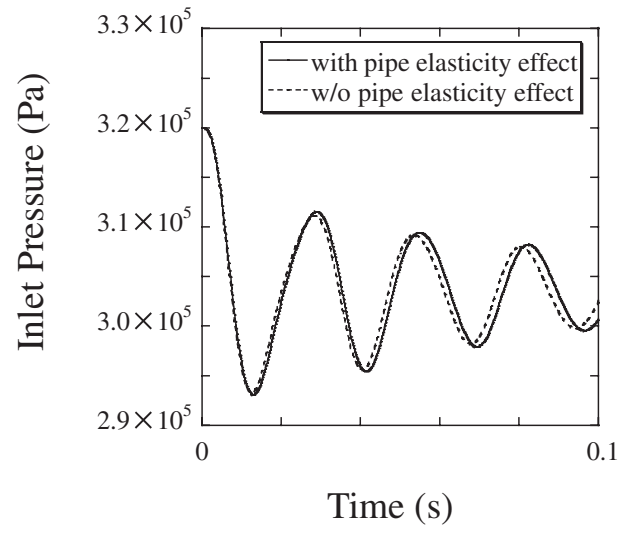

(a) Inlet pressure of $\mathrm{LH}_{2}$ feed system (compressible)

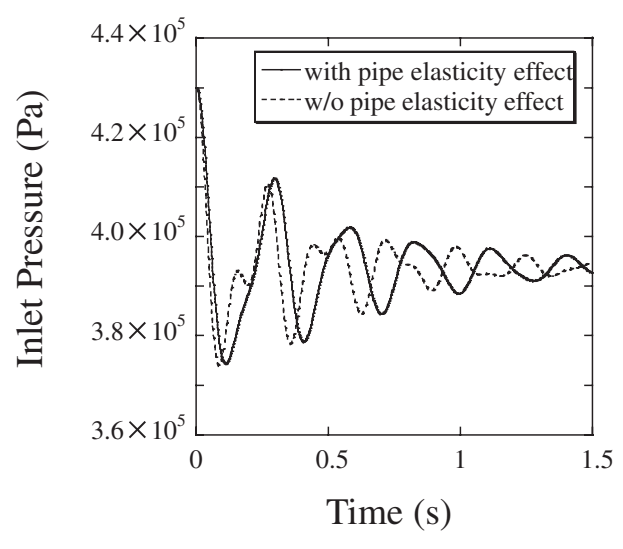

(b) Inlet pressure of LOX feed system (compressible)

Fig. 10. Effect of pipe elasticity $\left(5 \overline{C_{B}}, 0.1 \overline{M_{B}}\right)$.

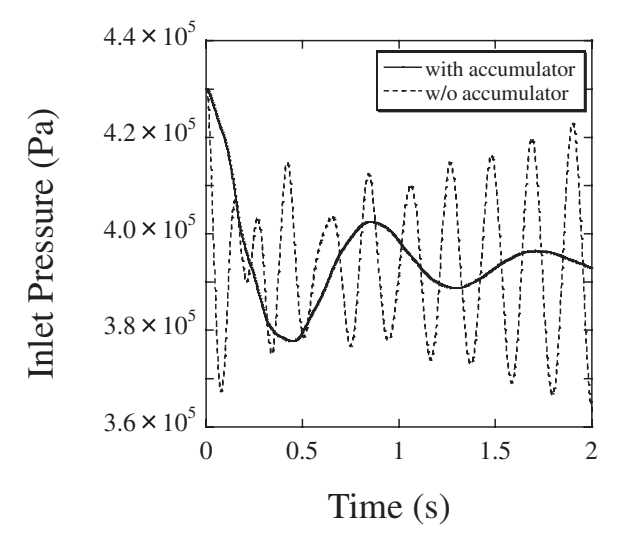

Fig. 11. Effect of an accumulator $\left(\overline{C_{B}}, 8 \overline{M_{B}}\right)$ (compressible).

and $M_{B}$ values of $1 \overline{C_{B}}$ and $8 \overline{M_{B}}$, respectively. By installing an accumulator in the inlet pipe of the LOX feed system, the response frequency of the inlet pressure becomes low and the system changes from unstable to stable. Obviously, the accumulator is a very effective device to suppress system surge and POGO instability.

\section{Conclusions}

The dynamic response characteristics of rocket pumps driving $\mathrm{LH}_{2}$ and $\mathrm{LOX}$ feed system were analyzed by a new numerical simulation utilizing the one-dimensional nonlinear compressible flow model. The main results are as follows:

1. For both compressible and incompressible models, cavitation compliance $\left(C_{B}\right)$ has a dominant effect on system response frequency and mass flow gain factor $\left(M_{B}\right)$ has a dominant effect on system stability.

2. The response frequency of the compressible model is less than that of the incompressible model and its amplitude decreases rapidly. These compressibility effects are more dominant in the $\mathrm{LH}_{2}$ feed system than in the LOX feed system.

3. For the compressible model, disturbance downstream of the pump is absorbed into the pump and does not greatly affect the flow upstream of the pump. This phenomenon is more dominant in the $\mathrm{LH}_{2}$ feed system than in the LOX feed system.

4. System response frequency becomes low when the effect of pipe elasticity is taken into consideration, an effect very similar to the effect of cavitation compliance.

5. An unstable system became stable by inserting an accumulator in the inlet pipe. Thus, an accumulator is a very effective device for suppressing cavitation surge and POGO instability.

\section{References}

1) Brennen, C. E. and Acosta, A. J.: The Dynamic Transfer Function for a Cavitating Inducer, ASME J. Fluid Eng., 98 (1976), pp. 182-191.

2) Brennen, C. E. and Acosta, A. J.: Theoretical, Quasi-Static Analysis of Cavitation Compliance in Turbopumps, J. Spacecraft Rockets, 10 (1973), pp. 175-180.

3) Brennen, C. E., Meissner, C., Lo, E. Y. and Hoffman, G. S.: Scale Effects in the Dynamic Transfer Functions for Cavitating Inducers, ASME J. Fluid Eng., 104 (1982), pp. 428-433.

4) Ng, S. L. and Brennen, C. E.: Experiments on the Dynamic Behavior of Cavitating Pumps, ASME J. Fluid Eng., 100 (1978), pp. 166-176.

5) Tsujimoto, Y., Kamijo, K. and Yoshida, Y.: A Theoretical Analysis of Rotating Cavitation in Inducers, ASME J. Fluid Eng., 115 (1993), pp. 135-141.

6) Tsujimoto, Y., Kamijo, K. and Brennen, C. E.: Unified Treatment of Flow Instabilities of Turbomachines, J. Propul. Power, 17 (2001), pp. 636-643.

7) Kamijo, K., Yoshida, M. and Tsujimoto, Y.: Hydraulic and Mechanical Performance of the LE-7 LOX Pump Inducer, J. Propul. Power, 9 (1993), pp. 819-826.

8) Shimura, T. and Kamijo, K.: Dynamic Response of the LE-5 Rocket Engine Liquid Oxygen Pump, J. Spacecraft Rockets, 22 (1985), pp. 195-200.

9) Shimura, T.: Geometry Effects in the Dynamic Response of Cavitating LE-7 Liquid Oxygen Pump, J. Propul. Power, 11 (1995), pp. 330-336.

10) Jun, S.-I., Tokumasu, T. and Kamijo, K.: Dynamic Response Analysis of a High Pressure $\mathrm{LH}_{2}$ Feed System, Turbomachinery, 31 (2003), pp. 459-466 (in Japanese).

11) BFT Experimental Data offered by courtesy of NASDA, 2003. 